\title{
Original Research \\ Novel tetrameric cell penetrating antimicrobial peptoids effective against mycobacteria and drug-resistant Staphylococcus aureus
}

\author{
Bettina Simone Fleck ${ }^{1,2, \dagger}$, Devika Mukherjee ${ }^{2, \dagger}$, Nhan Dai Thien Tram ${ }^{2}$, \\ Pui Lai Rachel Ee ${ }^{2, *}$, Ute Schepers ${ }^{1, *}$ \\ ${ }^{1}$ Institute of Functional Interfaces, Karlsruhe Institute of Technology (KIT), 76344 Eggenstein Leopoldshafen, Germany \\ ${ }^{2}$ Department of Pharmacy, National University of Singapore, 117544 Singapore, Singapore \\ *Correspondence: phaeplr@nus.edu.sg (Pui Lai Rachel Ee); Ute.schepers@kit.edu (Ute Schepers) \\ ${ }^{\dagger}$ These authors contributed equally. \\ Academic Editor: Melanie R. Power Coombs \\ Submitted: 30 October 2021 Revised: 1 January 2022 Accepted: 10 January 2022 Published: 14 February 2022
}

\begin{abstract}
Background: Antimicrobial peptides (AMPs) are short, cationic, amphipathic molecules that have gained tremendous popularity as alternatives to traditional antibiotics due to their lower propensity to develop bacterial resistance. However, the clinical developability of AMPs remains impeded due to shortcomings such as proteolytic instability and poor penetration leading to low bioavailability. Aims: To improve the access of AMPs to cells and subsequent bacteria killing, we evaluated the cell-penetrating and antimicrobial properties of three novel libraries of synthetic peptoids using Minimum Inhibitory Concentration, killing efficacy and membrane permeabilization assays against mycobacteria and Staphylococcus aureus. In addition, we investigated cell selectivity using mammalian cells to assess peptoid toxicity. Results: We showed that short tetrameric Rhodamine B-labeled peptoids composed of a balance of aromatic and lipophilic residues have potent selective antimicrobial activity against a range of microorganisms. The most potent candidates were active against drug-resistant $S$. aureus isolates as well as mycobacterial strains, with cell penetrating capabilities reported in HeLa and RAW 264.7 macrophage cells. Conclusions: These data suggest that peptoids with novel dual functionalities may potentially be an interesting class of therapeutics and/or molecular delivery agents for anti-infective purposes.
\end{abstract}

Keywords: peptoids; antimicrobial; cell penetrating; MRSA; Mycobacterium tuberculosis

\section{Introduction}

The unrestricted use of antibiotics over the past 50 60 years has created multidrug-resistant (MDR) strains of microorganisms for which currently only limited treatment options are available [1-5]. Compounding the problem, the development and approval of new antibiotics has not kept pace with the progression of this global health threat $[6,7]$. Based on reports from the U.S. Centers for Disease Control and Prevention (CDC), approximately 119,000 cases of $S$. aureus bloodstream infections occurred in 2017, resulting in significant number of deaths. In tandem, cases of both hospital and community-acquired methicillin-resistant S. aureus (MRSA) infections have not decreased in the past decade [8]. Other Gram-positive bacteria such as mycobacteria also account for a large number of community cases, especially in third world countries [7]. In 2018, 10 million people were diagnosed with tuberculosis (TB), with at least half a million of these new cases being rifampicin-resistant. Caused by the Mycobacterium tuberculosis bacteria, treatment regimens for drug-sensitive TB are complex involving long-term therapy over a period of 6 months with a cocktail of several drugs [7,9]. Hence, there is an urgent need for novel antimicrobial agents with new mechanisms of action that could replace or complement current conventional therapies.
Cationic antimicrobial peptides (AMPs) are a class of short, cationic, amphipathic molecules that constitute a major part of the innate host defense system in many organisms [10-13]. Their mechanism of action primarily involves the disruption or displacement of the bacterial membrane, giving rise to a lower likelihood of resistance development as compared to traditional antibiotics [13]. However, AMPs have several drawbacks including proteolytic instability and poor penetration leading to low bioavailability [12]. These shortcomings make the translation of AMPs as therapeutics extremely challenging. Oligo-Nsubstituted glycines, also known as antimicrobial peptoids (AMPos), are sequence-specific synthetic peptidomimetics with a peptide backbone but differ from AMPs in that the side chains are attached to the backbone amide nitrogen instead of the alpha-carbon (Fig. 1B) [14-16]. This structural difference means that there are no known proteases that will recognize and degrade the peptoid structure making them more stable. Peptoids also do not face similar conformational constraints as the corresponding peptides, due to the loss of chirality of the alpha-carbon. They are highly modifiable with diverse side chains, thus offering a toolkit for the development of artificial functional compounds $[17,18]$. There is increasing interest in peptoid development due to their antimicrobial activity against a broad 


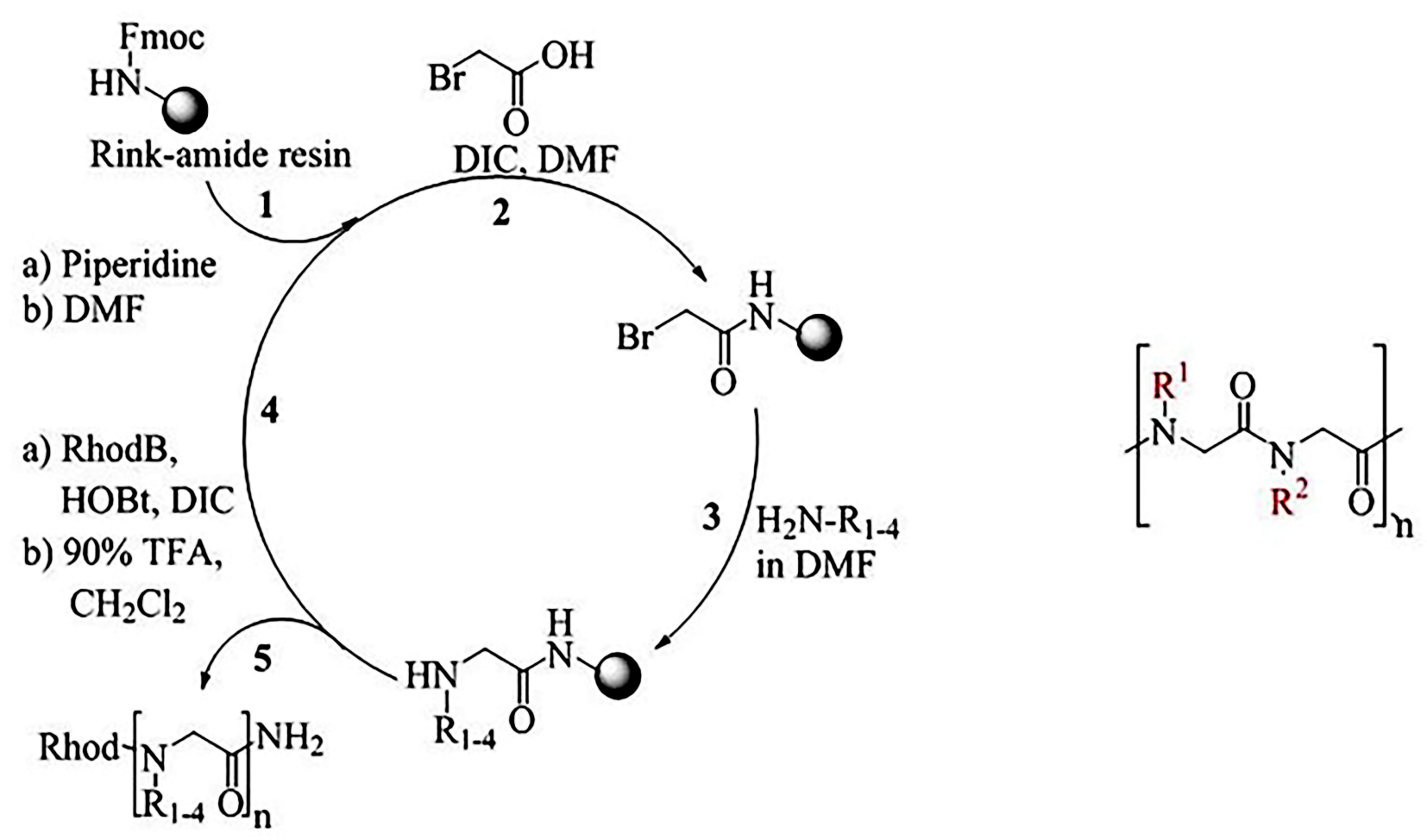

Fig. 1. Peptoid Synthesis. (A) Submonomer synthesis of peptoids. (B) General chemical structure of peptoids. Abbreviations: DIC, Diisopropylcarbodiimide; DMF, Dimethylformamide; RhodB, Rhodamine B; TFA, Trifluoroacetic acid. Sub-bullets a) and b) describe sub-sequential synthesis steps.

spectrum of pathogens, non-specific mode of action, decreased susceptibility to enzymatic degradation, and the relative ease of synthesis (Fig. 1A). The latter is also bolstered by the low costs of combinatorial solid phase synthesis [14]. More interestingly, peptoids have been shown to display better membrane permeability when compared to peptides, or by interacting with intracellular targets such as bacterial DNA.

As most AMPs resemble the amphiphilic properties of cell penetrating peptides or CPPs, their peptoid counterparts called Cell Penetrating Peptoids (CPPos) have been good mimics, despite the lack of chirality and absence of hydrogen bond formation by amide nitrogens. Multiple cationic charges favour endosomal accumulation, while lipophilicity promotes mitochondrial localization [19]. By using a radiofrequency tag supported combinatorial split and mix approach (IRORI), Kölmel et al. [17] showed that an increase in lipophilicity in tetrameric peptoids promoted their localization in mitochondria. By learning from mitochondrial CPPs and CPPos as described by Horton et al. [20] and Kölmel et al. [17], we developed a library of tetrameric cell penetrating antimicrobial peptoids with the suitable chemical properties for localization in mitochondria.
Three libraries comprising a total of 401 peptoids with varying side chains were designed and synthesized to study whether peptoids can have dual functionality of being both cell penetrating and antimicrobial. Our hypothesis was to examine whether the modulation of the lipophilicity of tetrameric peptoids would continue to allow for cell penetration without disrupting the cell membranes. These modifications would enable us to develop novel therapeutic peptoids with good biocompatibility and ease of synthesis.

\section{Materials and methods}

\subsection{Synthesis and purification of peptoid oligomers}

Peptoid synthesis was performed using IRORI technology at room temperature reported by Kölmel et al. [17] using permutation of four different side chains (Fig. 2). Library 1 was synthesized using $N$-(2-prop-2-yn 1 -yl) glycine ( $N$ prg), $N$-(p-chlorobenzyl)glycine ( $N$ pcb), $N$-(4aminobutyl)glycine, ( $N$ lys), and $N$-(benzyl)glycine ( $N$ phe) for side chains. Library 2 was performed with $N$ (benzyl)glycine ( $N$ phe), $N$-(1-aminotetradecanyl)glycine (Ntetradec), $N$-(4-Hydroxybenyl)glycine $(N \mathrm{phb})$ and $N$-(4fluorobenzyl) glycine $(N \mathrm{pfb})$ side chains. Library 3 was performed with $N$-(2-prop-2-yn-1-yl)glycine ( $N$ prg), $N$-(p- 
<smiles>[Y]C1CCC(C)C1</smiles>

Nprg

\section{Lipophilic}<smiles>CCN(CCCC[NH3+])CC(=O)C(C)C</smiles>

Nys

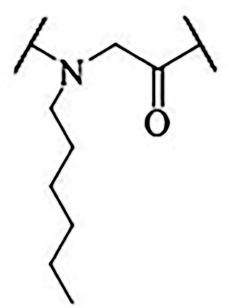

Whe

\section{$N$-(2-prop-2-yn-1-yl)glycine $\quad N$-(4-aminobutyl)glycine}<smiles>CCCCCCCCCCCCCCN(CC(=O)C(C)C)C(C)C</smiles>

Ntetradec

$N$-(tetradecyl)glycine

\section{$\underline{\text { Aromatic }}$}<smiles>CC(C)C(=O)CN(Cc1ccccc1)C(C)C</smiles>

Nphe

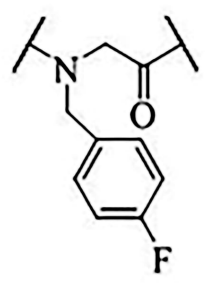

Npfb<smiles>CC(C)C(=O)CN(Cc1ccc(O)cc1)C(C)C</smiles>

Nphb<smiles>CC(C)C(=O)CN(Cc1ccc(Cl)cc1)C(C)C</smiles>

Npcb

\section{$N$-(benzyl)glycine $\quad N$-(4-fluorobenzyl)glycine $N$-(4-hydroxybenzyl)glycine $N$-(4-chlorbenzyl)glycine}

Fig. 2. Peptoid monomer side chain structures.

chlorobenzyl)glycine ( $N$ pcb), $N$-(4-Hydroxybenyl)glycine $(N \mathrm{phb})$ and $N$-(1-aminohexanyl)glycine (Nhe) side chains. All CPPos were labeled with Rhodamine B at the Cterminus of the peptoid as described in Kölmel et al. [17]. All products were verified to have $>95 \%$ purity by RPHPLC and lyophilized to dry powders prior to biological testing. Mass spectrometry was used to confirm the molecular weights of the purified products.

\subsection{Minimal inhibitory concentration (MIC) determination against $S$. aureus}

Minimum inhibitory concentration or MIC is the concentration at which a compound is able to inhibit the growth of bacteria. All experiments were conducted in microtiter plates. $75 \mu \mathrm{L}$ of $1 \times 10^{6} \mathrm{CFU} / \mathrm{mL}$ bacterial inoculum in Mueller-Hinton broth (MHB) was added to $75 \mu \mathrm{L}$ of peptoid solution in MHB (prepared in serial dilution). Negative control without peptoids was used while the positive control was performed with vancomycin treatment. The $\mathrm{MIC}_{90}$ was defined as the lowest concentration of peptoid that inhibited $90 \%$ of bacterial growth after incubation at $37^{\circ} \mathrm{C}$ for 18 hours at $150 \mathrm{rpm}$. Absorbance at $\mathrm{OD}_{600}$ was measured using a Tecan Infinite M2 to determine bacterial growth. MIC values reported were reproducible between three independent experimental replicates.

\subsection{MIC of mycobacteria}

MIC for mycobacteria was determined similar to $S$. aureus in a microtiter plate with minor modifications. $100 \mu \mathrm{L}$ of bacterial culture at a density of $5 \times 10^{6} \mathrm{CFU} / \mathrm{mL}$ were incubated with an equal volume of drug containing Middlebrook $7 \mathrm{H} 9$ media and incubated at $37^{\circ} \mathrm{C}$ for 5 days at $150 \mathrm{rpm}$. Bacterial growth was measured by absorbance $\left(\mathrm{OD}_{600}\right)$ similar to $S$. aureus. The negative control was a drug free culture which was considered as $100 \%$ growth rate. $90 \%$ growth was evaluated relative to the drug free wells. $0 \%$ growth was defined in wells containing only media without cultures.

\subsection{Octanol-water partition coefficient (cLogP)}

Molar attenuation coefficient of Rhodamine B in water and octanol at $\mathrm{I}=550 \mathrm{~nm}$ was determined by diluting Rhodamine B in octanol and water to a final concentration of $20 \mu \mathrm{M}$. The solution was measured in a 96-well-plate (Costar 3596, 96 Well Cell Culture Cluster, sterile), using a 96-well plate reader (Ultra Microplate Reader ELx808, BioTEK Instruments, INC). Peptoids were diluted to a final concentration of $160 \mu \mathrm{M}$ in $250 \mu \mathrm{L}$ water. Afterwards, 250 $\mu \mathrm{L}$ octanol was added and each mix was vortexed for $2 \mathrm{~min}$ and centrifuged for 3 min with a centrifugal force of 3000 $\mathrm{g}$ to separate the octanol from the aqueous phase. Phases were separated and the absorbance at I $=550 \mathrm{~nm}$ of 200 
Table 1. Molecular weight (MW), $\mathrm{MIC}_{90}$, and $\mathrm{cLogP}$ and of selected peptoid derivatives against drug resistant $\mathrm{S}$. aureus and mycobacteriad.

\begin{tabular}{|c|c|c|c|c|c|c|c|c|c|c|}
\hline \multirow[b]{2}{*}{ Peptoid } & \multirow[b]{2}{*}{ Sequence $^{a}$} & \multirow[b]{2}{*}{$\mathrm{MW}^{b} \mathrm{~g} / \mathrm{mol}$} & \multicolumn{7}{|c|}{$\mathrm{MIC}_{90}{ }^{c}(\mu \mathrm{M})$} & \multirow[b]{2}{*}{$\operatorname{cLog} \mathrm{P}$} \\
\hline & & & $\begin{array}{c}\text { S. aureus } \\
\text { ATCC 2973 }\end{array}$ & $\begin{array}{l}\text { S. aureus } \\
7 \text { DM4299 }\end{array}$ & $\begin{array}{l}\text { S. aureus } \\
\text { M0004583 }\end{array}$ & $\begin{array}{l}\text { S. aureus } \\
\text { DM1455 }\end{array}$ & $\begin{array}{l}\text { S. aureus } \\
\text { DR09808R }\end{array}$ & $\begin{array}{l}\text { M. bovis } \\
\text { BCG }\end{array}$ & 1. chelonae & \\
\hline 1 & $\begin{array}{l}\mathrm{H}-(N \text { phe- } N \text { prg- } N \text { pcb- } \\
\quad N \text { pcb })-\mathrm{NH}_{2}\end{array}$ & 1046 & 6.25 & 1.56 & $<1.56$ & $<1.56$ & 6.25 & 9.6 & 19.1 & 1.51 \\
\hline 2 & $\begin{array}{l}\mathrm{H}-(N \text { phe- } N \text { prg- } N \text { pcb- } \\
\quad N \text { phe })-\mathrm{NH}_{2}\end{array}$ & 1012 & 6.25 & 12.5 & 6.25 & 6.25 & 12.5 & 19.8 & $>148$ & 1.45 \\
\hline 3 & $\begin{array}{l}\mathrm{H}-(N \text { pfb- } N \text { pfb- } N \text { phe- } \\
\quad N \text { phe })-\mathrm{NH}_{2}\end{array}$ & 1067 & 1.56 & 6.25 & 3.12 & 3.12 & 6.25 & 18.8 & 70.3 & 1.52 \\
\hline 4 & $\begin{array}{c}\mathrm{H}-(N \text { pfb- } N \text { pfb- } N \text { pfb- } \\
N \text { pfb })-\mathrm{NH}_{2}\end{array}$ & 1103 & 1.56 & 3.12 & 3.12 & 3.12 & 3.12 & 18.1 & 34 & 1.49 \\
\hline 5 & $\begin{array}{c}\mathrm{H}-(N \mathrm{phb}-N \text { pfb- } N \text { phe- } \\
\quad N \text { pfb })-\mathrm{NH}_{2}\end{array}$ & 1083 & 1.56 & 6.25 & $<1.56$ & 3.12 & 3.12 & 9.2 & 34.6 & 1.19 \\
\hline 6 & $\begin{array}{c}\mathrm{H}-(N \mathrm{phb}-N \text { pfb- } N \text { phe- } \\
\quad N \text { phb })-\mathrm{NH}_{2}\end{array}$ & 1081 & 3.12 & 12.5 & 6.25 & 12.5 & 12.5 & 9.3 & 69.4 & 1.25 \\
\hline 7 & $\begin{array}{c}\mathrm{H}-(N \mathrm{phb}-N \mathrm{pfb}-N \text { pfb- } \\
\quad N \mathrm{pfb})-\mathrm{NH}_{2}\end{array}$ & 1101 & 1.56 & 12.5 & 3.12 & 6.25 & 6.25 & 18.2 & 68.1 & 1.14 \\
\hline 8 & $\begin{array}{c}\mathrm{H}-(N \text { he- } N \text { pcb- } N \text { pcb- } \\
\quad N \text { he })-\mathrm{NH}_{2}\end{array}$ & 1088 & 3.12 & 6.25 & 3.12 & 3.12 & 6.25 & 18.4 & 68.9 & 0.73 \\
\hline 9 & $\begin{array}{l}\mathrm{H}-(N \text { he- } N \text { pcb- } N \text { pcb- } \\
\quad N \text { phb })-\mathrm{NH}_{2}\end{array}$ & 1101 & 3.12 & 12.5 & 6.25 & 6.25 & 6.25 & 18.2 & 68.1 & 0.36 \\
\hline 10 & $\begin{array}{c}\mathrm{H}-(N \text { he- } N \text { he- } N \text { pcb- } \\
\quad N \text { prg })-\mathrm{NH}_{2}\end{array}$ & 1081 & 1.56 & 3.12 & $<1.56$ & $<1.56$ & 3.12 & 18.5 & 18.5 & 1.04 \\
\hline 11 & $\begin{array}{c}\mathrm{H}-(N \text { he- } N \text { he- } N \text { he- } \\
\quad N \text { prg })-\mathrm{NH}_{2}\end{array}$ & 951 & 1.56 & $<1.56$ & $<1.56$ & $<1.56$ & $<1.56$ & 21 & 21 & 1.41 \\
\hline
\end{tabular}

${ }^{a}$ See Fig. 2 for a guide to peptoid monomers.

${ }^{b} \mathrm{MW}$, Molecular weight.

${ }^{c} \mathrm{MIC}_{90}$ Minimum Inhibitory Concentration that inhibits $90 \%$ of bacterial growth.

${ }^{d}$ Maximum concentration tested $=200 \mu \mathrm{M}$ (the exception was M. bovis BCG and M. chelonae which was tested at $158 \mu \mathrm{M}$ ).

$\mu \mathrm{L}$ octanol phase and water phase was measured in a 96well-plate (Cstar 3596, 96 Well Cell Culture Cluster, sterile) by using a 96-well plate reader (Ultra Microplate Reader ELx808, BioTEK Instruments, INC). Each absorbance of peptoid was measured at least three times. Data were averaged and standard deviation was calculated.

\subsection{Live imaging by confocal microscopy}

Cell culture was carried out under sterile conditions. $1 \times 10^{4} \mathrm{HeLa}$ (human cervix carcinoma) cells or $2 \times 10^{4}$ RAW 264.7 cells were plated into each well of a 8 -well $\mu$ slide from IBIDI (Ibitreat, Martinsried, Germany) and cultured in $200 \mu \mathrm{L}$ Dulbecco's modified Eagle's medium, high glucose, supplemented with $10 \%$ Fetal calf serum and $1 \mathrm{U} / \mathrm{mL}$ penicillin/streptomycin at 37 ${ }^{\circ} \mathrm{C}, 5 \% \mathrm{CO}_{2}$. The purified peptoids were dissolved in distilled water to yield a $2 \mathrm{mM}$ stock solution and were further diluted with 10\% DMEM. The cells were incubated with the peptoids at a final concentration of $10 \mu \mathrm{M}$ at 37 ${ }^{\circ} \mathrm{C}, 5 \% \mathrm{CO}_{2}$. Cellular uptake of the peptoids was mea- sured by live-cell imaging after 24 hours. Visualization of the peptoids was achieved by confocal microscopy using a Leica TCS-SP5 II, equipped with a DMI6000 microscope (Germany). The peptoids were excited at $561 \mathrm{~nm}$ using a DPSS laser. The Mitotracker Green (Invitrogen, Karlsruhe, Germany) for the detection of the mitochondria was excited at $488 \mathrm{~nm}$ using an argon laser and the Hoechst 33342 for the detection of the nuclei was excited at 364 nm using a UV laser. The objective was an HCXPL APO CS $63.0 \times 1.2$ Water UV. The exposure was set to minimize oversaturated pixels in the final images. Fluorescence emission was measured at $417-468 \mathrm{~nm}$ for the detection of the nuclei, at 499-552 $\mathrm{nm}$ for the detection of the mitochondria, and 593-696 nm for the detection of the rhodamine B labelled peptoids. Image acquisition was conducted at a lateral resolution of $1024 \times 1024$ pixels and 8-bit depth using LAS-AF 2.0.2.4647 acquisition software (Leica LAS AF Lite 3.1, Leica Microsystems CMS GmbH, https://leica-las-af-lite.software.informer.com/4.0/). 


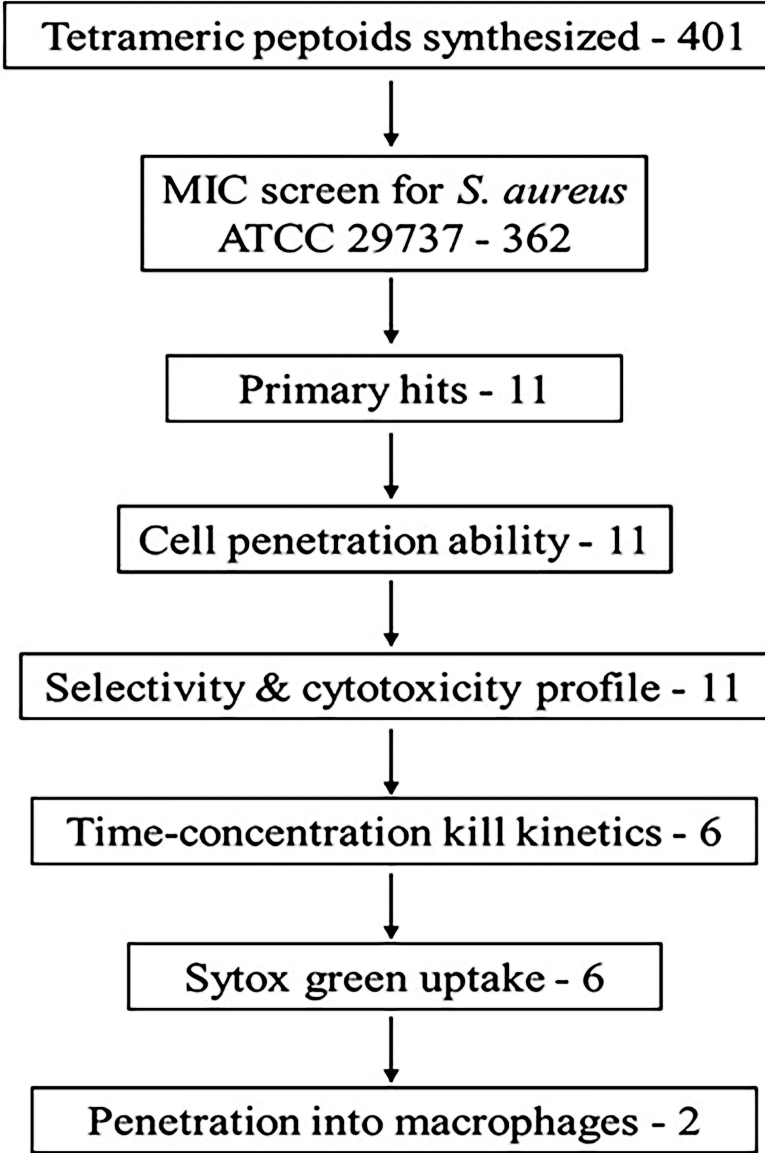

Fig. 3. Schematic of screening of hits and workup of lead compounds.

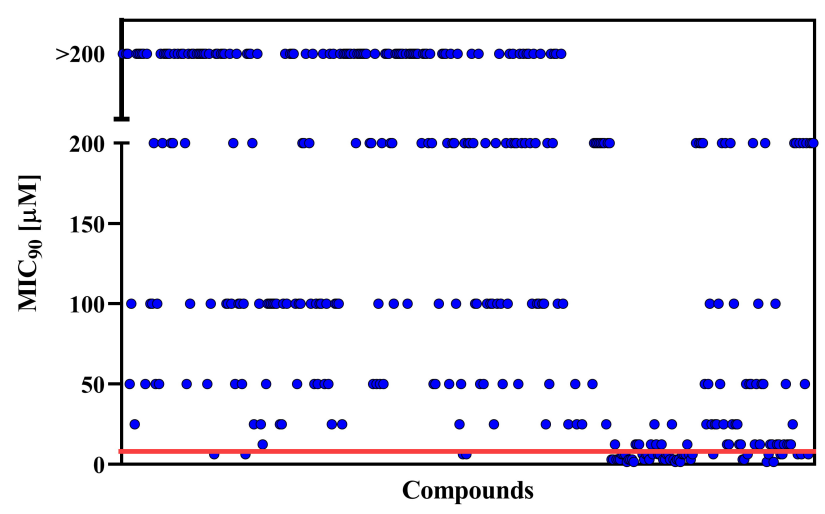

Fig. 4. Scatter plot of primary screen of peptoids against $\boldsymbol{S}$. aureus ATCC 29737. Three libraries, comprising a total of 362 compounds were screened for potency. A cut-off of $10 \mu \mathrm{M}$ was used as shown by the red line.

\subsection{Cell cytotoxicity assay}

HEK 293 T cells were cultured in DMEM media. A peptoid solution plate (100 $\mu \mathrm{L}$ per well) was prepared by serial dilution of peptoid stocks in media. Peptoid solution were transferred to a 96-well plate of day-old cell mono- layers containing $100 \mu \mathrm{L}$ per well media $\left(\approx 1 \times 10^{4}\right)$ cells per well. The plate was then incubated for 24 hours at 37 ${ }^{\circ} \mathrm{C}$ and $5 \% \mathrm{CO}_{2}$. MTS reagent (Promega) (40 $\mu \mathrm{L}$ per well) was added to each well, and the plate was incubated at 37 ${ }^{\circ} \mathrm{C}$ for 3 hours in darkness, after which absorbance at 490 $\mathrm{nm}$ was read. The percentage of inhibition was calculated as below.

Percentage of inhibition
$\times 100$

where $\mathrm{A}$ is the absorbance of the test well and $\mathrm{A}_{\text {control }}$ the average absorbance of wells with cells exposed to media

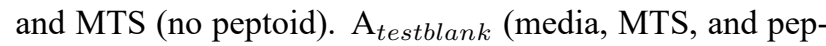
toid) and $\mathrm{A}_{\text {blank }}$ (media and MTS) were background absorbance measured in the absence of cells.

\subsection{Time concentration kill kinetics}

Peptoid solution $(100 \mu \mathrm{L})$ with concentrations corresponding to $1 \times, 2 \times$ and $4 \times$ MIC was first prepared and added to an equal volume of bacterial solution containing bacterial counts of approximately $10^{4} \mathrm{CFU} / \mathrm{mL}$ in each well of a 96-well plate. The plates were incubated at $37^{\circ} \mathrm{C}$ and samples were collected at different timepoints and plated on agar plates for determination of viable counts. The results were depicted as mean $\log (\mathrm{CFU} / \mathrm{mL}) \pm$ standard deviation.

\subsection{Membrane permeabilization study using flow cytometry}

Mid-log phase culture of $S$. aureus ATCC 29737 was diluted in fresh MHB to achieve an OD600 of 0.15. It was then washed twice with PBS and re-suspended in PBS. The suspension was incubated with $1 \times$ MIC of selected peptoids and $50 \mathrm{nM}$ SYTOX green for 2 hours at $37^{\circ} \mathrm{C}$. The incubation mixture was washed twice in PBS and resuspended in same volume of PBS. Flow cytometry was performed, and data was analysed using CytoFLEX LX Flow Cytometer and CytExpert (Beckman Coulter, Brea, CA, USA). The bacteria suspension was also incubated with $35 \%$ ethanol and $50 \mathrm{nM} \mathrm{SYTOX}$ green for 1 hour at $37^{\circ} \mathrm{C}$ as the positive control. Controls of unstained cells, and peptoid treatment in the absence of SYTOX green were also analysed to account for background noises. Values reported were reproducible between three independent experimental replicates using ANOVA tests.

\section{Results and discussion}

\subsection{Peptoid design and synthesis}

We synthesized libraries of AMPos to determine whether the antimicrobial activities of peptoids are affected by structural and sequence modifications in a similar fashion as AMPs [21,22]. The design of the peptoids in these libraries was derived from changes in lipophilicity and amphiphilicity. Sequence-specific N-substituted glycine oligomers can be efficiently synthesized via pri- 

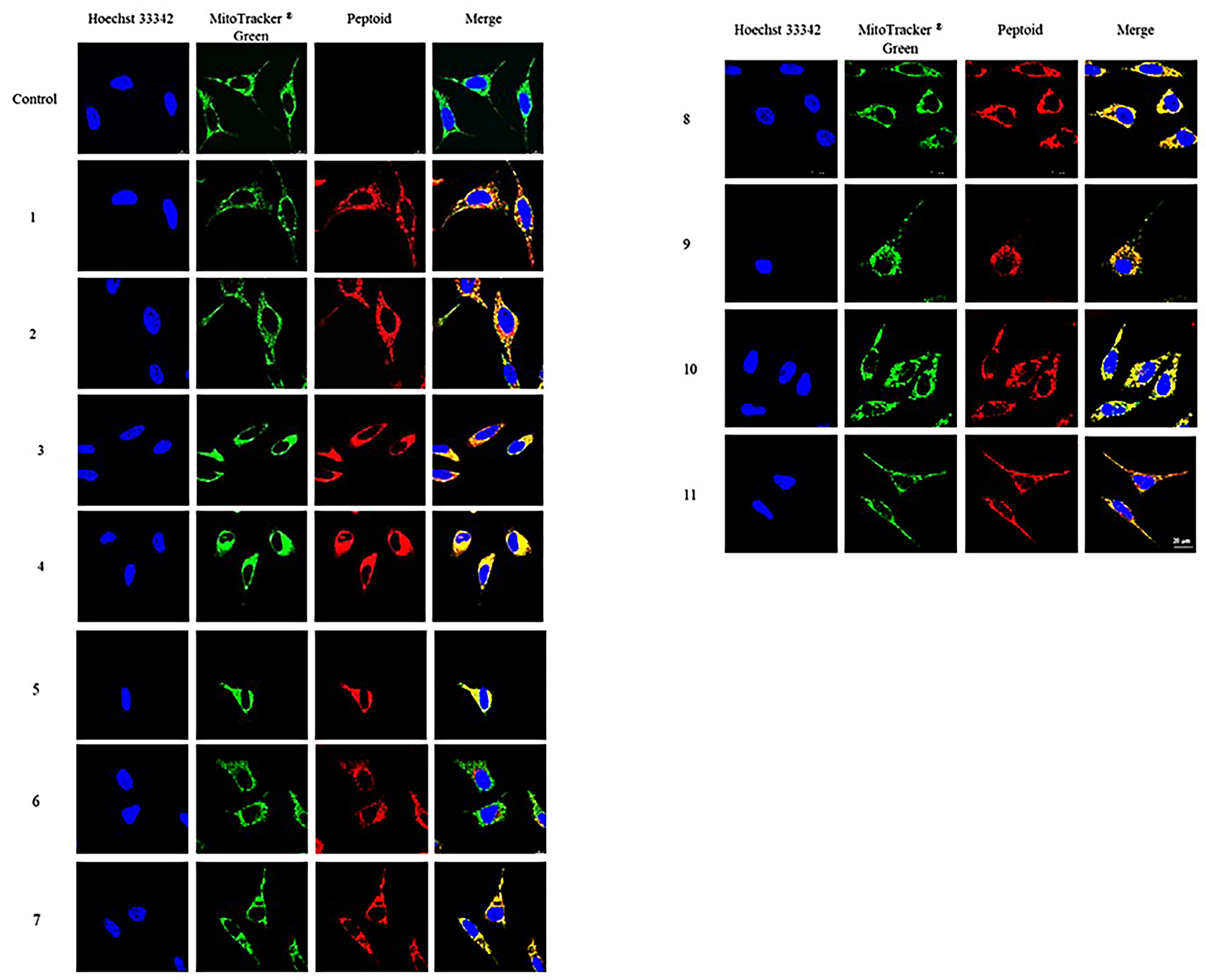

Fig. 5. Cellular uptake of library 1 (peptoid 1, 2), library 2 (peptoid 3, 4, 5, 6, 7, 8) and library 3 (8, 9, 10, 11) compounds in HeLa cells. $1 \times 104 \mathrm{HeLa}$ cells were treated with $10 \mu \mathrm{M}$ of peptoid tagged with Rhodamine B for 24 hours at $37^{\circ} \mathrm{C}$. For co -staining of the nuclei and the mitochondria the cells were treated with Hoechst 33342 (blue) and $100 \mathrm{nM}$ MitoTracker Green (green). Eventually the cells were analyzed by fluorescence confocal imaging. The last column shows the merges of the respective emission channels of each line by using the following PMTs for the emission: 417-468 nm for the detection of the nuclei (blue), 499-552 nm for the detection of the mitochondria (green), and 593-696 nm for the detection of the rhodamine B labelled peptoids (red). Scale bar $=20 \mu \mathrm{m}$.

mary amines as submonomers to incorporate a large number of diverse side chain functionalities [14]. This method iterates sequential steps of bromoacylation and nucleophilic displacement of bromide using any kind of amine primary amine submonomer synthons. $\mathrm{N}$-acetylated linear oligomers were synthesized on the Rink amide resin to obtain C-terminal amides. Thereafter, the $N$ - termini were acetylated with acetic anhydride prior to TFA cleavage (Fig. 1A). In total, 401 peptoids were synthesized using split mix combinatorial solid-phase synthesis. Amines used for synthesis in Library 1 were 4-aminobutylglycine (Nlys), prop-2-yn-1-amine ( $N$ prg), benzylamine ( $N$ phe) and 4 chlorobenzylamine $(N \mathrm{pcb})$. Amines used for synthesis in Library 2 are tetradecylglycine ( $N$ tetradec), 4 fluorobenzylglycine $(N \mathrm{pfb})$, benzylamine ( $N$ phe) and 4hydroxybenzylglycine (Nphb). Amines used for synthesis in Library 3 are hexylamine (Nhe), prop-2-yn1-amine ( $N$ prg), 4-hydroxybenzylglycine ( $N$ phb) and 4chlorobenzylamine $(N \mathrm{pcb})$. For microscopic analysis, all peptoids have been labelled with Rhodamine B. All other experiments have also been performed with labelled peptoids. The monomers used for the synthesis are shown in Fig. 2. The positive charge was introduced by incorporating lysine-like (Nlys) monomers, while the overall lipophilicity was altered using a variety of short and aromatic monomers. The chemical structures for the side chains used in creating the libraries were mimics of natural amino acid side chains, in addition to a broader selection of bulky hydrophobic side 

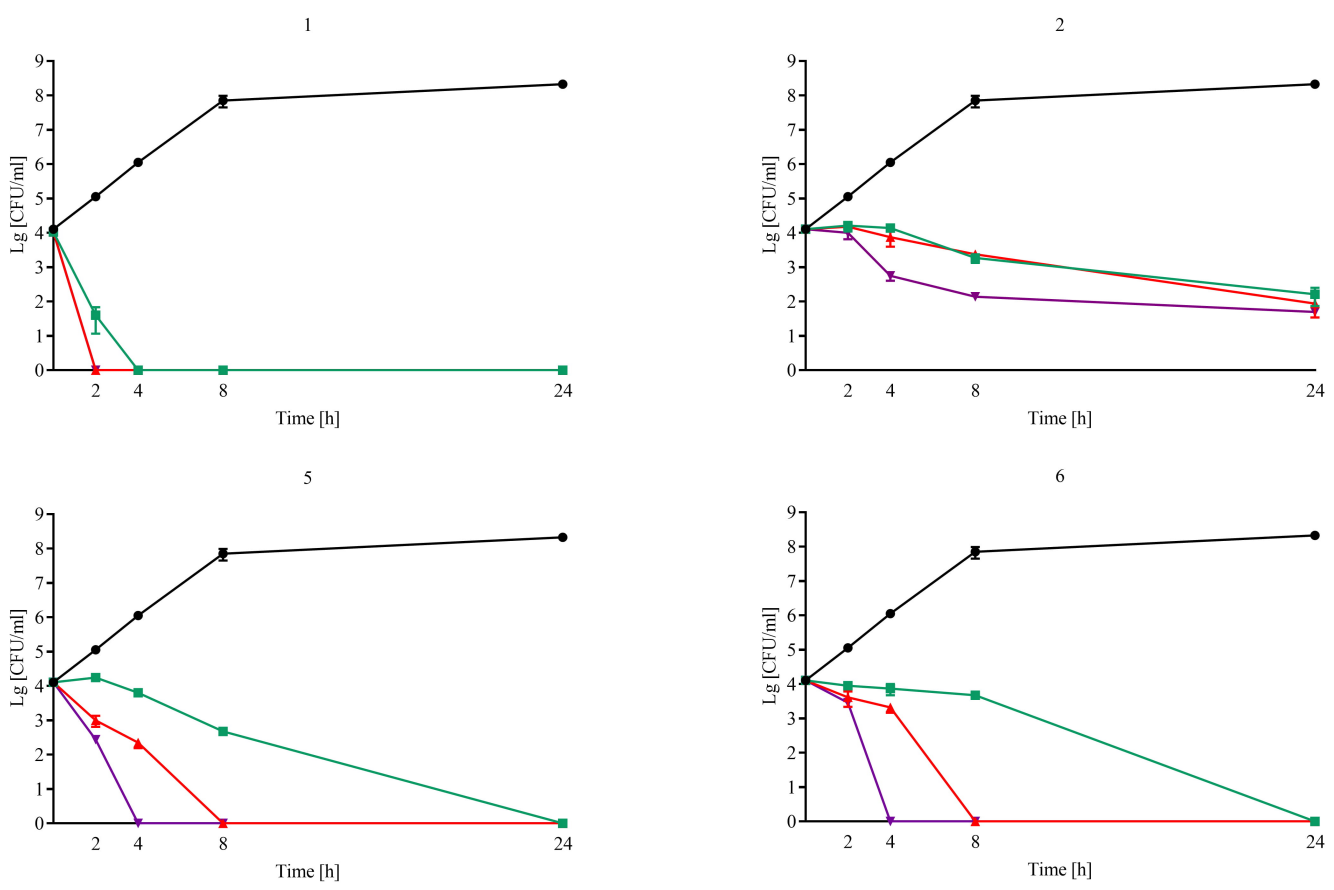

10
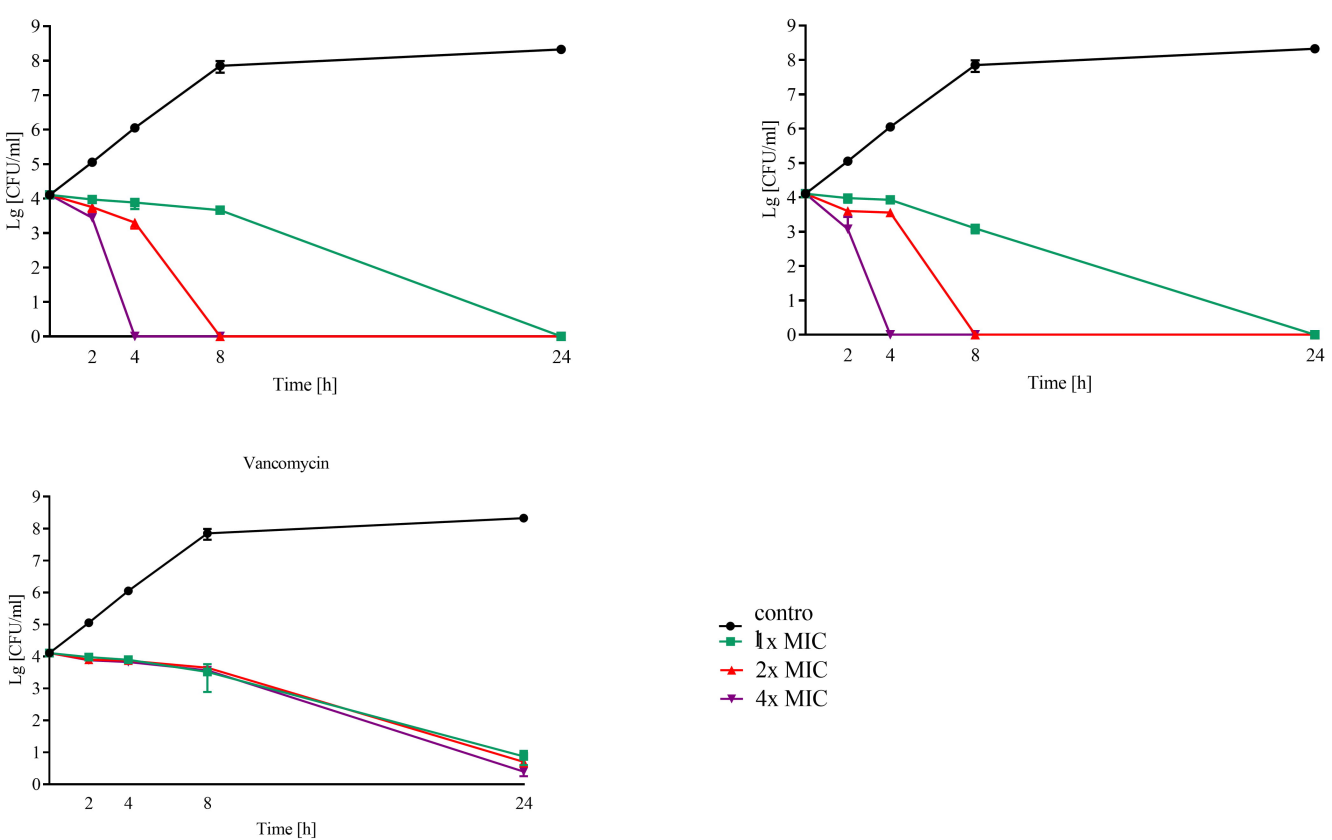

contro

$\div 1 \times \mathrm{MIC}$

$\pm 2 \times$ MIC

* 4x MIC

Fig. 6. Time-concentration kill kinetics of peptoids and Vancomycin against $\boldsymbol{S}$. aureus ATCC 29737. Vancomycin was used as a positive control. The CFU/mL values represent the means \pm standard deviations from two independent experiments.

chains such as $N \mathrm{phb}, N \mathrm{pfb}$, and $N \mathrm{pcb}$. To vary the amphiphilicity of the peptoids, we used different permutations of the monomers in the peptoid sequence.

\subsection{Screening cascade}

401 compounds were synthesized, of which 362 were screened for $\mathrm{MIC}_{90}$ against S. aureus ATCC 29737 (Fig. 3). 11 compounds were identified as primary hits with $\mathrm{MIC}_{90}$ of $<10 \mu \mathrm{M}$ which were then observed for cell penetration properties in HeLa cells. They were then tested for antimicrobial activity against mycobacteria and drug-resistant $S$. aureus clinical isolates. Their selectivity between mammalian and bacterial cells was then determined by evaluating cytotoxicity against mammalian cell lines. Six peptoids were chosen for determining time concentration kill kinetics, as well as uptake of SYTOX Green to establish whether membrane permeabilization was indeed the mechanism of action. Finally, three molecules, one as a representative 


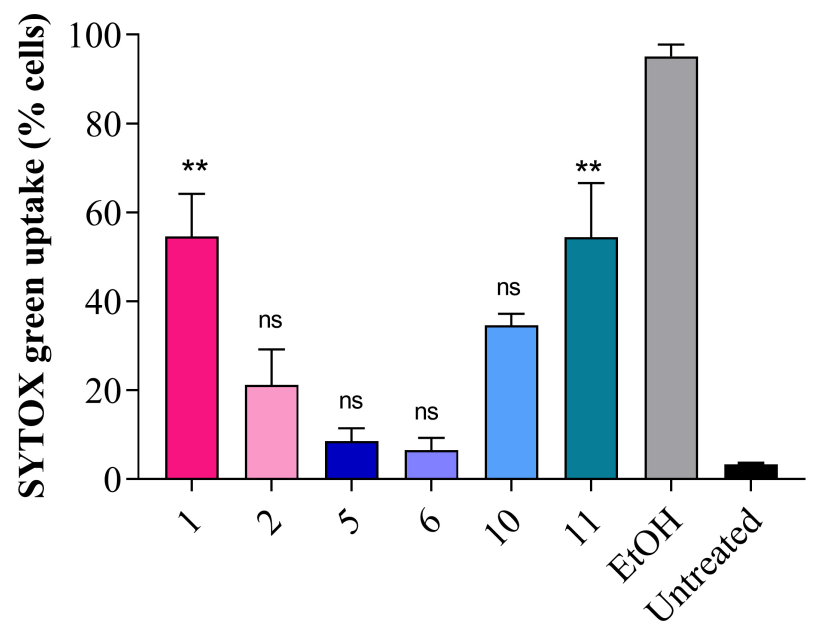

Fig. 7. Membrane permeabilization of $S$. aureus ATCC 29737 induced by the peptoids, as reflected by the percentage of cells in the culture positive for SYTOX green uptake.

from each library, were tested for uptake by macrophages as both mycobacteria and $S$. aureus can be localized inside mammalian macrophages. This would also enable us to determine whether cellular uptake was dependent on the cell type.

\subsection{Antibacterial activity}

362 peptoids were screened for their antimicrobial activity against $S$. aureus ATCC 29737 (Fig. 4). The highest tested concentration was $200 \mu \mathrm{M}$. The potent molecules were identified as those with an MIC of $<10 \mu \mathrm{M}$, as denoted by the red cut-off line on the graph. MIC values for compounds with no activity even at $200 \mu \mathrm{M}$ were denoted as $>200 \mu \mathrm{M}$. The potent peptoids were then tested against drug-resistant clinical isolates of $S$. aureus, Mycobacterium bovis (M. bovis) BCG (as a BSL-2 substitute of $M$. tuberculosis) and Mycobacterium chelonae (M. chelonae) (as a representative of non-tuberculous mycobacteria). Of the peptoids screened, those with activity against all tested strains are summarized in Table 1.

For the initial MIC screening, S. aureus was incubated with different concentrations of peptoids for 18 hours. MICs were determined by measuring OD $_{600}$. The MIC was defined as the concentration that can inhibit $90 \%$ of bacterial growth $\left(\mathrm{MIC}_{90}\right)$. The active peptoids with $\mathrm{MIC}<10$ $\mu \mathrm{M}$ were then screened against the mycobacterial species and Escherichia coli (E. coli) (data not shown) to determine whether they were potential antimicrobials. Interestingly, we found no activity against $E$. coli which led us to conclude that these peptoids are selectively active only against Gram-positive bacteria and mycobacteria which are known to be closely related to Gram positive bacteria [23]. Recent studies have discovered that hydrophobicity is strongly suggested to enhance selective activity of antimicrobial peptides (AMPs) against Gram-positive bacte- ria due to the insertion of peptides into Gram-positive membranes while weakening their ability to penetrate Gramnegative outer membrane [24-26]. Membrane components such as charged lipopolysaccharides as present on Gramnegative bacteria outer membranes present additional electrostatic barriers to peptide and peptoid entry. It is likely that our active peptoids with similar membrane penetrative abilities as AMPs are unable to penetrate the Gram-negative $\mathrm{OM}$ as easily as they did for Gram-positive bacteria. Table 1 summarizes the sequences of the active peptoids, MICs against the different strains of bacteria and the cLogP value which denotes the lipophilicity of the molecules. The cLogP value was in the range between 0.36 and 1.52 .

The lowest $\mathrm{MICs}_{90}$ were within the range $1.5 \mu \mathrm{M}$ and $12.5 \mu \mathrm{M}$. It has been shown in literature that compounds containing fluorine and chlorine incorporated in them generally show high antimicrobial activity [27,28]. Here, we examined whether the inclusion of fluorine and chlorine can enhance the antimicrobial activity of our peptoids. All peptoids demonstrated a decent antimicrobial effect ( $\mathrm{MIC}_{90}$ $1.56 \mu \mathrm{M}$ to $6.25 \mu \mathrm{M}$ ) against $S$. aureus ATCC 29737 with peptoid 1, 10, and 11 being effective against both strains of mycobacteria. Moreover, all shortlisted peptoids exhibited a potent activity ( $\mathrm{MIC}_{90} 1.56 \mu \mathrm{M}$ to $12.5 \mu \mathrm{M}$ ) against MDR $S$. aureus. The higher MICs in mycobacteria compared to $S$. aureus can be attributed to the differences in the cell wall structure of the two bacterial species. Mycobacteria are known to have an outer layer of mycolic acid making them relatively more impermeable to antibiotics, thus harder to kill [23].

\subsection{Cell penetrating peptoids}

Cell membranes are known to be selectively permeable and hence limit the penetration of large molecules or non-lipophilic molecules such as hydrophilic and large molecular weight drugs [17,29]. As a result, antimicrobials are unable to reach intracellular targets or intracellular microbes. This in turn restricts the arsenal of drugs that can be employed to efficiently target intracellular microbes [30]. Existing studies have shown the use of viral vectors as shuttle for AMPs or membrane disruption for delivery, but this can potentially result in unintended cytotoxicity. Since peptoids have previously been shown to overcome barriers for cell penetration without membrane destruction, our libraries of peptoids were similarly tested for these abilities. Hela cells have been widely used in several studies to determine the cell penetrative ability of peptides [31,32]. Due to their robust proliferative capability, we have chosen them as hosts for large scale screening of our peptoids in this study. It has also been established that most of the peptide entry mechanisms are endocytic in nature and do not involve specific cell receptors, hence HeLa cells though not perfect will act as good surrogates for determining the cell penetrating ability of our peptoids. Here, we showed that all our peptoids except for peptoid 6, localized in the 
mitochondria. We used the Pearson correlation coefficient $(\mathrm{Rr})$ to estimate the co-localization in the mitochondria, using the ImageJ Plugin JACoP (ImageJ bundled with 64bit Java, ImageJ, https://imagej.nih.gov/ij/download.html, https://imagej.net/plugins/jacop). All peptoids showed a coefficient of $0.75-0.95$, except for peptoid $6(0.43)$ which in this case could indicate endosomal localization rather than mitochondrial entry. The $\mathrm{Rr}$ for the peptoids were as follows: $\operatorname{Rr}(1)=0.75, \operatorname{Rr}(2)=0.824, \operatorname{Rr}(3)=0.917, \operatorname{Rr}$ $(4)=0.957, \operatorname{Rr}(5)=0.869, \operatorname{Rr}(6)=0.438, \operatorname{Rr}(7)=0.911$, $\operatorname{Rr}(8)=0.922, \operatorname{Rr}(9)=0.916, \operatorname{Rr}(10)=0.944, \operatorname{Rr}(11)=$ 0.909 . This is confirmed by the confocal images in Fig. 5 which showed yellow colour in the merged column except for peptoid 6. This indicates that all the peptoids except 6 localized in the mitochondria making them ideal for targeting intracellular pathogens or even as delivery agents to transport antimicrobial agents into cells.

\subsection{Evaluation of selectivity}

We evaluated the biocompatibility of selected oligomers in HEK 293T cells by using the MTS assay. $\mathrm{CC}_{50}$ was defined as the concentration that is cytotoxic to $50 \%$ of the cells. The cytotoxicity was estimated using a tetrazolium salt (MTS)-based colorimetric assay and is reported as the concentration that inhibits metabolic activity by $50 \%\left(\mathrm{CC}_{50}\right)$. We defined the selectivity ratio (SR) as the quotient of $\mathrm{CC}_{50}$ and the $\mathrm{MIC}_{90}$ S. aureus ATCC 29737, hence the SR is an estimate of a compounds' tendency to kill bacterial rather than mammalian cells. Table 2 summarizes the $\mathrm{MIC}_{90}$ for $S$. aureus ATCC 29737, $\mathrm{CC}_{50}$ and SR. Peptoid 5, 6 and 7 showed lower toxicities to HEK 293T cells and the highest selectivity with an SR of 32. High selectivity ratios represent peptoids which preferentially kill bacterial rather than mammalian cells.

Table 2. Antimicrobial activity against $S$. aureus ATCC 29737, CC $_{50}$ of HEK 293T cells and SR.

\begin{tabular}{lccc}
\hline Peptoid ID & $\begin{array}{c}\mathrm{MIC}_{90}(\mu \mathrm{M}) \\
\text { S. aureus }\end{array}$ & $\mathrm{CC}_{50}{ }^{a}(\mu \mathrm{M})$ & $\mathrm{SR}^{b}$ \\
\hline 1 & 6.25 & 12.5 & 2 \\
2 & 6.25 & $>100$ & 16 \\
3 & 1.56 & 25 & 16 \\
4 & 1.56 & 12.5 & 8 \\
5 & 1.56 & 50 & 32 \\
6 & 3.122 & $>100$ & $>32$ \\
7 & 1.56 & 50 & 32 \\
8 & 3.122 & 25 & 8 \\
9 & 3.122 & 25 & 8 \\
10 & 1.56 & 25 & $>16$ \\
11 & 1.56 & 12.5 & 8 \\
\hline
\end{tabular}

${ }^{a} \mathbf{C C}_{50}$, concentration that results in death of $50 \%$ of cells.

${ }^{b} \mathbf{S R}$, Selectivity Ratio (SR) $=\mathrm{CC}_{50} / \mathrm{MIC}_{90}$ S. aureus ATCC 29737.

\subsection{Killing efficiency}

We then investigated the ability of the peptoids 1,2 , 5, 6, 10, and 11 to effectively kill S. aureus ATCC 29737 as shown in Fig. 6. Peptoid 1 had a killing efficiency of $99.99 \%(>3-\log )$ at $1 \times$ MIC at $4 \mathrm{~h}$. Peptoid 2 reduced the bacterial load by $2-\log$, however, the activity was only timedependent and not dose-dependent as increasing the concentration to $2 \times$ and $4 \times$ did not affect the efficacy. The killing efficiency of peptoids 5, 6, 10 and 11 was found to be $99.99 \%(>3-\log )$ at $2 \times$ MIC at $8 \mathrm{~h}$. Furthermore, at $4 \times$ MIC, there was complete eradication of all bacteria after 4 $\mathrm{h}$ incubation respectively. On the other hand, vancomycin, which is an existing drug used to treat $S$. aureus infections, was ineffective in killing $99.99 \%$ of the bacteria within 24 $\mathrm{h}$ and did not show dose dependent activity. Thus, we were able to demonstrate efficient bactericidal activity against $S$. aureus to be both time- and dose-dependent for all the peptoids except for peptoid 2.

\subsection{Flow cytometry}

To examine whether the selected peptoids caused membrane permeabilization of $S$. aureus ATCC 29737, cultures were treated with $1 \times$ MIC of the peptoids for 2 hours. Flow cytometry was employed to estimate the portion of bacterial population with permeabilized membranes. Membrane activity of test compounds was determined by measuring the uptake of SYTOX Green, a fluorescent dye which can only enter cells with compromised membrane integrity and stain intracellular nucleic acids. During flow cytometry analysis, this dye allows for differentiation between the bacterial cells which have been permeabilized and those which are intact. As shown in Fig. 7, compared to the untreated control, treatment with all selected peptoids resulted in SYTOX Green uptake of a portion of the cultures. However, the percentage of damaged cells varied with the different peptoids. Whereas peptoids 1 and 11 permeabilized the membrane of more than half of the bacterial population (54.6\% and 54.4\% respectively), membrane activity of peptoids 2 and 10 were less prominent $(21.2 \%$ and $34.7 \%$ respectively). Peptoids 5 and 6 barely affected the membrane, causing less than $10 \%$ of the culture to become permeable to SYTOX Green. Combining these results together with the time-concentration kill kinetics and the net charge of the peptoids, we can conclude that for peptoids 10 and 11 membrane disruption occurred before the bactericidal activity. This results in lysis leading to cell death. Peptoid 1 is cytotoxic as seen by the low selectivity index. It is likely that Peptoid 1 lysed cell membranes, bacterial or mammalian, extensively thus allowing uptake of SYTOX Green and rapid bactericidal activity. Peptoid 2, 5 and 6 exhibited a low uptake of SYTOX Green indicating that the membrane is intact. They remain, however, bactericidal as seen from the time kill kinetics, implying that there may be more than one mechanism of action involved in the activity of these peptoids. 


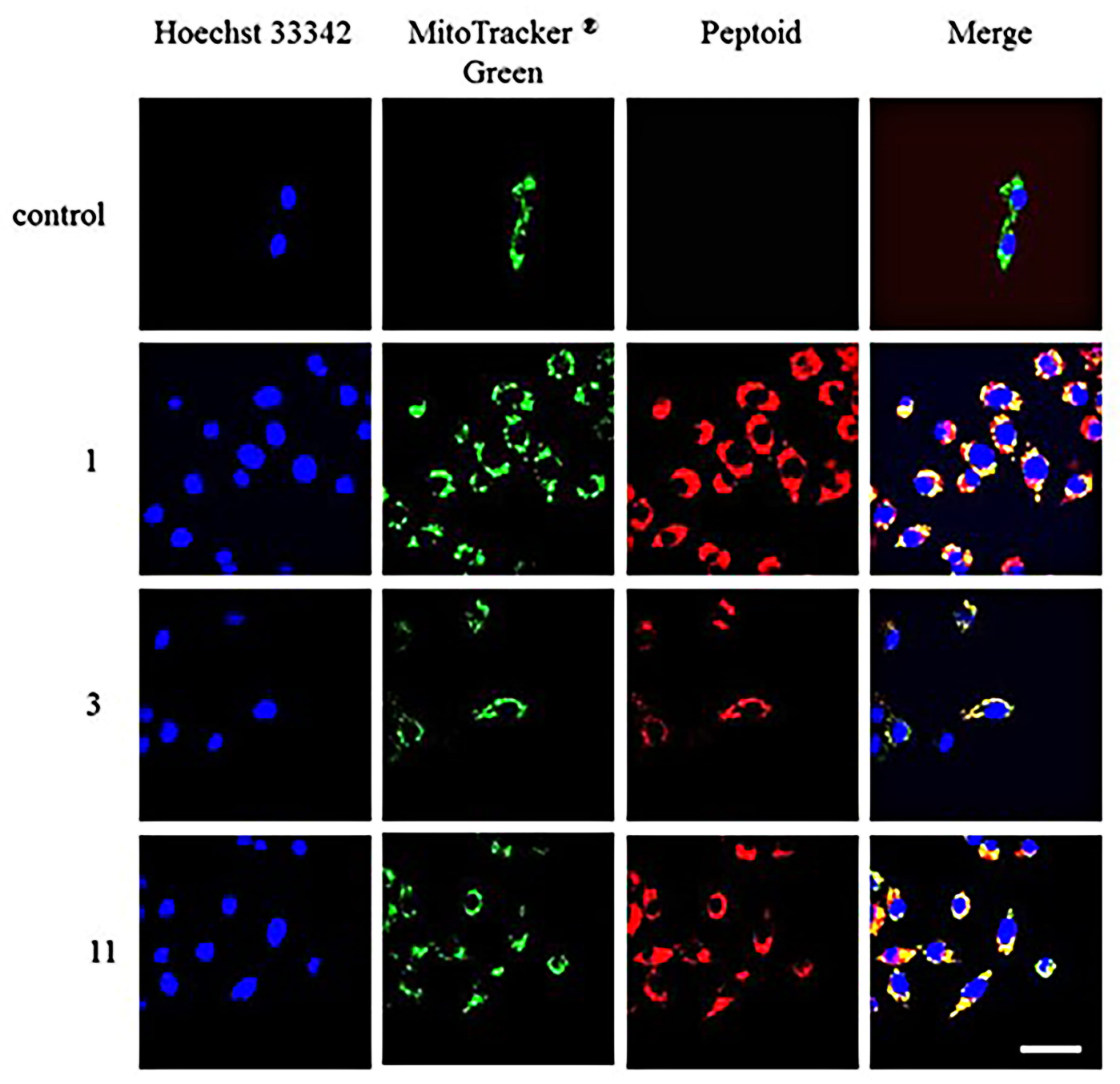

Fig. 8. Cellular uptake of peptoid 1, 3, 11 RAW 264.7 cells. $2 \times 10^{4}$ RAW 264.7 cells were treated with $10 \mu \mathrm{M}$ of peptoid tagged with Rhodamine B for 24 hours at $37{ }^{\circ} \mathrm{C}$. For co-staining of the nuclei and the mitochondria the cells were treated with Hoechst 33342 (blue) and $100 \mathrm{nM}$ Mitochondria GreenTM (green). Eventually, the cells were analyzed by fluorescence confocal imaging. The last column shows the merges of the respective emission channels of each line by using the following PMTs for the emission: 417-468 nm for the detection of the nuclei (blue), 499-552 nm for the detection of the mitochondria (green), and 593-696 nm for the detection of the rhodamine B labelled peptoids (red). Scale bar $=20 \mu \mathrm{m}$.

\subsection{Localisation in macrophages}

Granuloma, which are compact aggregates of immune cells such as macrophages, are the hallmark structures of tuberculosis. It is historically regarded as a host-protective structure that shields off the infected area from healthy tissue [33]. This is problematic as most drugs cannot penetrate the granuloma, and thus cannot reach the inside-residing bacteria. As a result, the bacteria can become dormant with the potential of future reactivation, especially when patients become immune-compromised [34]. Thus, it is important that our peptoids are able to penetrate into macrophages to exert their antimicrobial function. We incubated RAW 264.7 cells with peptoid solution ( 1 representative peptoid from each library) (labelled with Rhodamine B) and imaged the localization of the peptoids in living cells using confocal microscopy (Fig. 8). Cellular uptake in macrophages was detected for the selected peptoids. The confocal microscopy images showed, that the peptoids accumulated in the mitochondria of the cells. This finding provides a strong justification to the design of cell penetrating peptoids which can enter and localize within macrophages. This suggests that our peptoids could be a good starting point for development of new antimycobacterial drugs or as adjunctive therapy in combination with existing regimens.

\section{Conclusions}

Here we have demonstrated that short tetrameric Rhodamine B-labeled peptoids composed of a mix of aromatic 
and lipophilic residues have potent antimicrobial activity and are selective towards bacterial cells. The peptoids demonstrated activity against drug resistant $S$. aureus isolates as well as mycobacterial strains. Further, we demonstrated the cell penetrating capability of potent leads in HeLa and RAW 264.7 cells with their localization in the mitochondria. These data suggest that these labelled peptoids with novel dual functionalities at its best will act as interesting tools for the further study of short tetrameric antimicrobial peptoids as a novel class of therapeutics and/or molecular delivery agents for anti-infective purposes. Further studies are underway to examine the peptoids at their pristine states for in vivo efficacy.

\section{Author contributions}

BSF and DM contributed equally to this work. BSF, DM and NDTT conducted the experiments. BSF, DM, US and PLRE conceptualized this work and designed the experiments. BSF and DM analysed the data and prepared the figures. BSF, DM, US and PLRE wrote the manuscript. All authors have approved the final version of the manuscript.

\section{Ethics approval and consent to participate}

Not applicable.

\section{Acknowledgment}

We would like to thank Dr Lakshminarayanan Rajamani, Singapore Eye Research Institute for the drug resistant clinical isolates of $S$. aureus. B.S.F. acknowledges the support of the Jürgen Manchot Stiftung and the German Academic Exchange Service (DAAD). N.T. was awarded the NUS Research Scholarship. We acknowledge support by the KIT-Publication Fund of the Karlsruhe Institute of Technology.

\section{Funding}

This work was supported by the Deutsche Forschungsgemeinschaft (DFG), within the Research Training Group 2039 (B.S.F. and U.S.) and the Helmholtz Program Biointerfaces in Techology and Medicine (BIF-TM). U.S. and S.B. acknowledge funding by the Deutsche Forschungsgemeinschaft (DFG, German Research Foundation) under Germany's Excellence Strategy via the Excellence Cluster 3D Matter Made to Order (EXC-2082/1 - 390761711). This research is supported by the Singapore Ministry of Health's National Medical Research Council under its Individual Research Grant Scheme (NMRC/OFIRG/0026/2016 awarded to E.P.L.R.).

\section{Conflict of interest}

The authors declare no conflict of interest.

\section{References}

[1] Spellberg B, Powers JH, Brass EP, Miller LG, Edwards JE. Trends in Antimicrobial Drug Development: Implications for the Future. Clinical Infectious Diseases. 2004; 38: 1279-1286.

[2] Peschel A, Sahl HG. The Co-Evolution of Host Cationic Antimicrobula Peptides and Microbial Resistance. Nature Reviews Microbiology. 2006; 4: 529-536.

[3] World Health Organization. Antimicrobial resistance: global report on surveillance. 2014. Available at: https://apps.who.int/iri s/handle/10665/112642 (Accessed: 1 June 2021).

[4] Yang JH, Bening SC, Collins JJ. Antibiotic efficacy-context matters. Current Opinion in Microbiology. 2017; 39: 73-80.

[5] Aslam B, Wang W, Arshad MI, Khurshid M, Muzammil S, Rasool MH, et al. Antibiotic resistance: a rundown of a global crisis. Infection and Drug Resistance. 2018; 11: 1645-1658.

[6] Frieri M, Kumar K, Boutin A. Antibiotic resistance. Journal of Infection and Public Health. 2017; 10: 369-378.

[7] World Health Organization. Global Tuberculosis Report. 2019. Available at: https://www.who.int/publications/i/item/ 9789241565714 (Accessed: 1 June 2021).

[8] Kourtis AP, Hatfield K, Baggs J, Mu Y, See I, Epson E, et al. Vital Signs: Epidemiology and Recent Trends in MethicillinResistant and in Methicillin-Susceptible Staphylococcus aureus Bloodstream Infections - United States. Morbidity and Mortality Weekly Report. 2019; 68: 214-219.

[9] Glickman MS, Jacobs WR. Microbial pathogenesis of Mycobacterium tuberculosis: dawn of a discipline. Cell. 2001; 104: $477-$ 485.

[10] Khara JS, Priestman M, Uhía I, Hamilton MS, Krishnan N, Wang $\mathrm{Y}$, et al. Unnatural amino acid analogues of membrane-active helical peptides with anti-mycobacterial activity and improved stability. The Journal of Antimicrobial Chemotherapy. 2016; 71: 2181-2191.

[11] Khara JS, Wang Y, Ke X, Liu S, Newton SM, Langford PR, et al. Anti-mycobacterial activities of synthetic cationic $\alpha$-helical peptides and their synergism with rifampicin. Biomaterials. 2014; 35: 2032-2038.

[12] Hancock REW, Sahl H. Antimicrobial and host-defense peptides as new anti-infective therapeutic strategies. Nature Biotechnology. 2006; 24: 1551-1557.

[13] Zasloff M. Antimicrobial peptides of multicellular organisms. Nature. 2002; 415: 389-395.

[14] Zuckermann RN, Kerr JM, Kent SBH, Moos WH. Efficient Method for the Peparation of Peptoids [oligo(N-substituted glycines)] by Submonomer Solid-Phase Synthesis. Journal of the American Chemical Society. 1992; 114: 10646-10647.

[15] Miller SM, Simon RJ, Ng S, Zuckermann RN, Kerr JM, Moos WH. Comparison of the Proteolytic Susceptibilities of Homologous L-Amino Acid, D-Amino Acid, and N-Substituted Clycine Peptide and Peptoid Oligomers. Drug Development Research. 1995; 35: 20-32.

[16] Khara JS, Mojsoska B, Mukherjee D, Langford PR, Robertson $\mathrm{BD}$, Jenssen $\mathrm{H}$, et al. Ultra-Short Antimicrobial Peptoids Show Propensity for Membrane Activity Against Multi-Drug Resistant Mycobacterium tuberculosis. Frontiers in Microbiology. 2020; 11: 417.

[17] Kölmel DK, Fürniss D, Susanto S, Lauer A, Grabher C, Bräse $\mathrm{S}$, et al. Cell Penetrating Peptoids (CPPos): Synthesis of a Small Combinatorial Library by Using IRORI MiniKans. Pharmaceuticals. 2012; 5: 1265-1281.

[18] Vollrath SBL, Fürniss D, Schepers U, Bräse S. Amphiphilic peptoid transporters-synthesis and evaluation. Organic \& Biomolecular Chemistry. 2013; 11: 8197-8201.

[19] Kölmel DK, Hörner A, Rönicke F, Nieger M, Schepers U, Bräse S. Cell-penetrating peptoids: introduction of novel cationic side 
chains. European Journal of Medicinal Chemistry. 2014; 79: 231-243.

[20] Horton KL, Stewart KM, Fonseca SB, Guo Q, Kelley SO. Mitochondria-penetrating peptides. Chemistry \& Biology. 2008; 15: 375-382.

[21] Bessalle R, Kapitkovsky A, Gorea A, Shalit I, Fridkin M. AllD-magainin: chirality, antimicrobial activity and proteolytic resistance. FEBS Letters. 1990; 274: 151-155.

[22] Chongsiriwatana NP, Patch JA, Czyzewski AM, Dohm MT, Ivankin $\mathrm{A}$, Gidalevitz $\mathrm{D}$, et al. Peptoids that mimic the structure, function, and mechanism of helical antimicrobial peptides. Proceedings of the National Academy of Sciences of the United States of America. 2008; 105: 2794-2799.

[23] Brown L, Wolf JM, Prados-Rosales R, Casadevall A. Through the wall: extracellular vesicles in Gram-positive bacteria, mycobacteria and fungi. Nature Reviews Microbiology. 2015; 13: 620-630.

[24] Malanovic N, Lohner K. Antimicrobial Peptides Targeting Gram-Positive Bacteria. Pharmaceuticals. 2016; 9: 59.

[25] García AB, Viñuela-Prieto JM, López-González L, Candel FJ. Correlation between resistance mechanisms in Staphylococcus aureus and cell wall and septum thickening. Infection and Drug Resistance. 2017; 10: 353-356.

[26] Tram NDT, Selvarajan V, Boags A, Mukherjee D, Marzinek J, Cheng B, et al. Manipulating turn residues on de novo designed b-hairpin peptides for selectivity against drug resistant bacteria. Acta Biomaterialia. 2021; 135: 214-224.

[27] Mendoza-Figueroa HL, Serrano-Alva MT, Aparicio-Ozores G,
Martínez-Gudiño G, Suárez-Castillo OR, Pérez-Rojas NA, et al. Synthesis, antimicrobial activity, and molecular docking study of fluorine-substituted indole-based imidazolines. Medicinal Chemistry Research. 2018; 27: 1624-1633.

[28] Noszticzius Z, Wittmann M, Kály-Kullai K, Beregvári Z, Kiss I, Rosivall L, et al. Chlorine dioxide is a size-selective antimicrobial agent. PLoS ONE. 2013; 8: e79157.

[29] Sebbage V. Cell-Penetrating Peptides and their Therapeutic Applications. Bioscience Horizons. 2009; 2: 64-72.

[30] Marouseau E, Neckebroeck A, Larkin H, Le Roux A, Volkov L, Lavoie CL, et al. Modular sub-monomeric cell-penetrating guanidine-rich peptoids - synthesis, assembly and biological evaluation. RSC Advances. 2017; 7: 6059-6063.

[31] Patel SG, Sayers EJ, He L, Narayan R, Williams TL, Mills EM, et al. Cell-penetrating peptide sequence and modification dependent uptake and subcellular distribution of green florescent protein in different cell lines. Scientific Reports. 2019; 9: 6298.

[32] Foerg C, Ziegler U, Fernandez-Carneado J, Giralt E, Rennert R, Beck-Sickinger AG, et al. Decoding the entry of two novel cellpenetrating peptides in HeLa cells: lipid raft-mediated endocytosis and endosomal escape. Biochemistry. 2005; 44: 72-81.

[33] Ramakrishnan L. Revisiting the role of the granuloma in tuberculosis. Nature Reviews Immunology. 2012; 12: 352-366.

[34] Mukherjee D, Zou H, Liu S, Beuerman R, Dick T. Membranetargeting am-0016 kills mycobacterial persisters and shows low propensity for resistance development. Future Microbiology. 2016; 11: 643-650. 